\title{
Occupational and Patient Radiation Dose and Quality Implications of Femoral Access Imaging During Coronary Angiography
}

Kelly Wilson-Stewart, (D) ${ }^{1,2}$ Davide Fontanarosa, ${ }^{3,4}$ Eva Malacova, ${ }^{3,5}$ Steven Gett, ${ }^{2}$ Allan Kruger, ${ }^{2,6}$ Jamie V Trapp'

'School of Chemistry and Physics, Faculty of Science, Queensland University of Technology, Brisbane, QLD, 4000, Australia;

${ }^{2}$ Cardiiovascular Suites, Greenslopes Private Hospital, Brisbane, QLD, 4I20, Australia; ${ }^{3}$ School of Clinical Sciences, Faculty of Health, Queensland University of Technology, Brisbane, QLD, 4000, Australia; ${ }^{4}$ Centre for Biomedical Technologies (CBT), Queensland University of Technology, Brisbane, QLD, 4000, Australia; ${ }^{5}$ QMIR Berghofer Medical Research Institute, Herston, QLD, 4006, Australia; ${ }^{6}$ Department of Vascular Surgery, Royal Brisbane and Women's Hospital, Herston, QLD, 4029, Australia

Video abstract

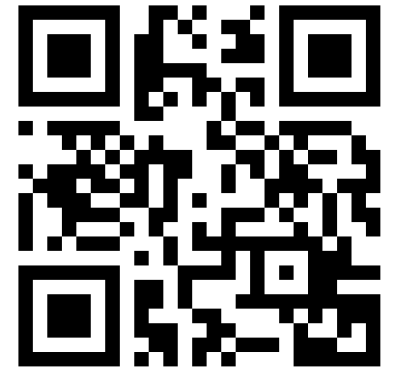

Point your SmartPhone at the code above. If you have a $Q R$ code reader, the video abstract will appear. Or use: https://youtu.be/fkaiBXXpPsE

Correspondence: Kelly Wilson-Stewart Cardiovascular Suites, Greenslopes Private Hospital, Newdegate Street, Greenslopes, Brisbane, QLD, 4120, Australia

Tel +6I 733947962

Fax +6I 733947969

Email ks.stewart@hdr.qut.edu.au
Purpose: Cardiologists often perform angiography of the common femoral artery (CFA) access site to evaluate whether the anatomy is suitable for deployment of a vascular closure device or to assess whether iatrogenic vessel damage has occurred. The choice of acquisition mode has radiation dose implications. The objective of this study was to investigate the influence of the selected type of CFA x-ray imaging mode (fluoro save, cine acquisition and digital subtraction angiography (DSA)) and tube angle on patient and staff dose during coronary angiography.

Materials and Methods: Assessment of image quality for the different modes was performed to determine whether lower dose modes provide images of sufficient clinical quality to be routinely employed. Radiation dose levels for the patients $(n=782)$, cardiologists $(n=17)$, scrub nurses $(n=27)$ and scout nurses $(n=32)$ were measured in a prospective singlecentre study between February 2017 and August 2019. Three Philips angiographic units and DoseAware dose monitoring systems were used.

Results: Among the acquisition modes, fluoro save provided acceptable diagnostic quality for visualizing femoral access points and diagnosing pathology in $99 \%$ of cases. Average patient dose area product (DAP) was $83.95,742.50$, and $3363.41 \mathrm{mGy}^{2}$ and average patient air kerma (AK) was $0.87,8.44$, and $18.61 \mathrm{mGy}$ for fluoro save, cine, and DSA acquisitions, respectively. The use of higher dose imaging modes, imaging in the contralateral view and utilizing steeper TA was associated with a higher patient dose. Due to staff dose being highly correlated with DAP and AK, it was difficult to observe any association between staff dose and CFA imaging mode. However, this does not discount a potential increase in occupational dose due to the use of cine angiography or digital subtraction angiography during CFA imaging.

Conclusion: DSA of the CFA should be avoided during transfemoral coronary angiography unless critical to diagnostic analysis. It is recommended that fluoroscopic operators consider utilizing lower dose modes in the ipsilateral orientation $\leq 32^{\circ}$ TA to reduce the risk of patient and staff radiation exposure.

Keywords: occupational exposure, cardiac catheterization, vascular closure device, patient dose, theater nurse, $\mathrm{x}$-ray imaging

\section{Plain Language Summary}

Cardiac angiography is an $\mathrm{x}$-ray procedure that allows cardiologists to image and treat pathology within a patient's coronary arteries. The heart arteries can be accessed via the common femoral artery (CFA) in the groin. The use of radiation during these procedures may result in adverse tissue effects to both the patient and the staff involved in the procedure. 
It is routine for the cardiologist to perform angiography of the CFA access site, and they have the choice of three imaging modes. Fluoroscopic imaging, which is the lowest dose mode, can be retrospectively stored, cine angiography is approximately 10 times the dose of fluoroscopy, and digital subtraction angiography is 100 times the dose of fluoroscopy.

This study sought to determine what the additional dose implications were in using higher dose modes for both the patient, and the staff involved in procedures, and whether the additional dose could be justified by improved diagnostic image quality. The effect of the angle of the x-ray tube on dose was also investigated.

We determined that the lowest dose mode provided images of sufficient quality to provide relevant clinical information, with significantly less dose to the patient. The x-ray tube angle was also found to have a significant effect on patient dose.

Due to staff dose being highly correlated with patient dose, it was difficult to observe any association between staff dose and CFA imaging mode, but this does not discount a potential increase in occupational dose due to the use of higher dose imaging modes.

DSA of the CFA should be avoided during transfemoral coronary angiography unless critical to diagnostic analysis. It is recommended that fluoroscopic operators consider utilizing lower dose modes with the recommended tube orientation and angles $\leq 32^{\circ}$ to reduce the risk of patient and staff radiation exposure.

\section{Introduction}

Cardiac angiography is a procedure that allows cardiologists to image and treat pathology within a patient's coronary arteries. X-rays are utilized during the examination to visualize radiographic contrast within the vessels. With an exponential increase in the number and complexity of catheter-based coronary procedures over the last decade, the concern over radiation exposure to the patient and the staff involved in procedures has also increased. ${ }^{1,2}$ The potentially deleterious tissue effects of protracted occupational exposure to low-dose ionizing radiation are not well understood, ${ }^{3}$ but there may be an increased risk of developing cancer, ${ }^{4,5}$ circulatory diseases, ${ }^{6}$ or chromosomal aberrations. ${ }^{7}$ A strong association between occupational exposure and the incidence of posterior subcapsular opacities in occupationally exposed personnel has been demonstrated. ${ }^{1,8}$

The heart arteries are accessed by introducing a catheter either via the radial artery (RA) in the arm or the common femoral artery (CFA) in the groin. While access via the radial artery is increasingly utilized in modern practice, CFA access remains a necessity in some patients. $^{1,8}$

It is routine for the cardiologist to perform angiography of the CFA access site to evaluate whether the anatomy is suitable for deployment of a vascular closure device (VCD) or whether iatrogenic vessel damage has occurred ${ }^{9}$.

VCDs have the benefit of accelerated hemostasis and prompt patient ambulation and have also been shown to reduce the risk of vascular complications. ${ }^{10}$ The choice of acquisition modes is fluoro save, cine angiography, which has an increased dose of 10-50 times higher than fluoro save, ${ }^{11,12}$ or digital subtraction angiography which may result in a dose 100 times that of fluoro save fluoroscopy. ${ }^{12}$

The use of pre-procedural femoral angiography can reduce complication rates by $40 \%,{ }^{9}$ and may be performed with the x-ray tube anterior-posterior (AP), in the ipsilateral (IL) oblique (eg, the right anterior oblique [RAO] for the right CFA), or the contralateral (CL) oblique (eg, the left anterior oblique [LAO] for the right CFA). ${ }^{11}$ The selected tube angle has also been demonstrated to effect both patient and staff dose. ${ }^{13,14}$

The choice of $x$-ray tube angle and imaging sequences may be determined by departmental protocol, intended purpose, and operator preference. One argument for the use of higher dose acquisitions is the superior quality provided by cine or DSA as opposed to fluoroscopic imaging. To the best of the author's knowledge, there is currently no literature examining the effect of femoral imaging mode and tube angle on patient and staff dose during coronary angiography. Additionally, there are no published studies appraising whether imaging CFA access points with higher dose modes provides enhanced diagnostic capabilities. Pearl et al performed phantom experiments to investigate the effect of dose modes during cerebral angiography. Results demonstrated that when using a straight tube angle, there was a 95\% reduction in patient DAP when using fluoro save compared to DSA. ${ }^{15}$

This study had three aims, with the primary aim being to investigate the influence of the CFA imaging mode on staff (Aim 1) and patient dose (Aims 1 and 2) during femorally accessed coronary angiography. The effect of femoral imaging tube angle (TA) on patient dose was also investigated (Aim 2). Additionally, image quality of differing x-ray acquisitions (including the potential influence exerted by patient BMI) was also considered to determine whether lower dose modes provide images of sufficient clinical quality to assess suitability for VCD deployment and diagnose pathology (Aim 3). 
The underlying premise of using ionizing radiation for imaging is to keep the exposure As Low as Reasonably Achievable (ALARA), and operators should select the mode with the lowest dose acceptable for the clinical task. ${ }^{13}$ It is hoped that this study will provide evidence to underpin clinical choices regarding CFA imaging angle and mode.

\section{Materials and Methods}

\section{Patient and Staff Dose (Aim I)}

Radiation dose levels for the patients $(n=782)$, cardiologists $(n=17)$, scrub nurses $(n=27)$ and scout nurses $(n=32)$ were measured in an observational prospective single-centre study. Data were collected for interventional and diagnostic coronary angiograms performed in three dedicated fluoroscopic rooms at a large Australian tertiary hospital between February 2017 and August 2019. All rooms were fitted with Philips Allura Xper equipment with two of the rooms installed with Clarity dose reducing software (Philips Healthcare, Best, Netherlands) and were more frequently used. A fluoroscopic rate of 15 frames per second (fps) was used, and cine and DSA acquisitions were taken at 15 and 4 fps, respectively. The patient dose parameters of air kerma (AK) and dose area product (DAP) were recorded for each case. Procedural characteristics such as total fluoroscopy time, number of cine runs, contrast volume, plaque complexity, and patient body mass index (BMI) were also collected.

Staff exposure was prospectively measured and downloaded via DoseAware badges (Philips Healthcare, Best, Netherlands) worn near the left temple, which in the absence of dedicated eye dosimeters provides an acceptable alternative to measure estimated eye dose. ${ }^{16}$ The majority of nursing staff chose to utilize lead (or lead equivalent) skull caps and glasses, whereas most cardiologists preferred to wear lead glasses only. Badges were attached externally to protective apparel (Figure 1). Lead coats, thyroid shields, and lead shin pads were consistently worn by staff. All rooms were equipped with both table-mounted and ceiling mounted lead shields as demonstrated in Figure 2. An additional lead shield was occasionally utilized by the scout nurse when situated on the non-shielded side of the patient table. Only cases performed via the CFA were included, and aborted procedures or workups for transcatheter aortic valve implants were excluded. Approval was granted by the Ramsay Human Research Ethics Committee (Protocol number-16/67) and informed, written consent was obtained from staff participants. As all identifying information was removed prior to analysis, patient consent was deemed unnecessary by the ethics committee. All components of this study were conducted in accordance with the Declaration of Helsinki.

\section{Patient Dose per Discrete CFA Imaging Event (Aim 2)}

Patient DAP and AK measurements were recorded for discrete CFA imaging events $(n=754)$ (independent of the data utilized in Aim 1). All images were acquired on equipment utilizing dose reducing software. The dose levels during fluoro save, cine angiography and DSA was compared. Differences in dose when using IL, CL imaging
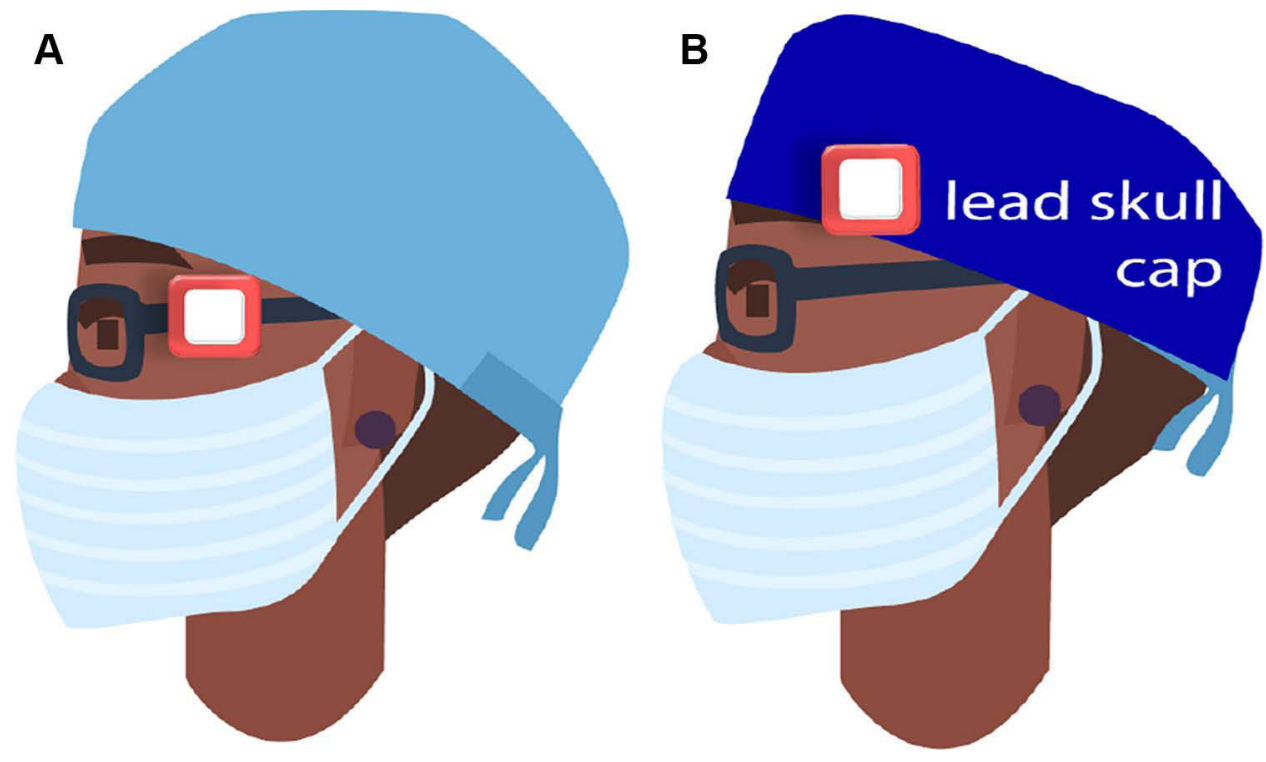

Figure I DoseAware dosimeter placement on the left temple external to lead glasses (A) and skull cap (B). 


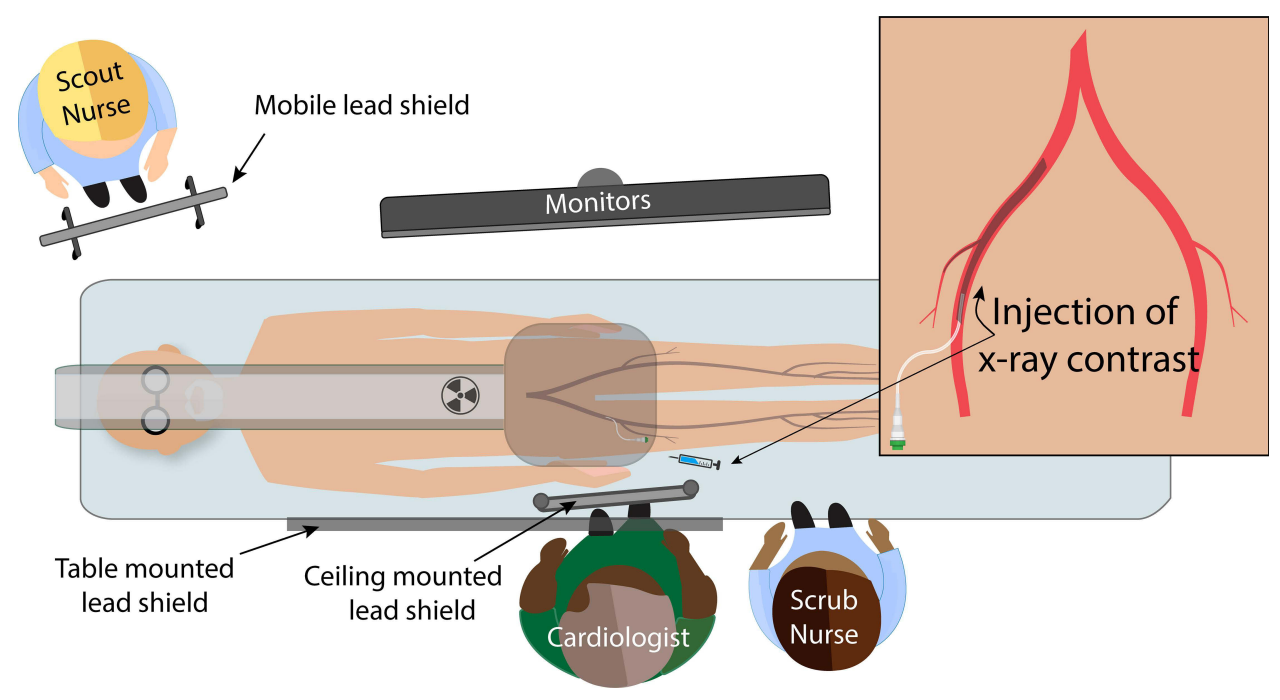

Figure 2 Typical room setup and staff location when imaging the femoral artery during coronary angiography.

angles, and the effect of TA on patient dose were investigated. TA was categorized as demonstrated in Figure 3. The most frequently utilized tube angles were between 22 and 32 degrees and were hence allocated as moderate.

\section{Image Quality Assessment (Aim 3)}

Two vascular surgeons with over 20 years' experience in surgical and catheter-based interventions were recruited to assess image quality via questionnaire. Fluoro save, cine, and DSA acquisitions $(\mathrm{n}=131)$ of the CFA during 106 coronary angiography procedures were retrospectively selected and randomly ordered. Procedural information was removed, and images were evaluated on a single dedicated medical-grade 19 inch liquid crystal display viewing station with an optimum resolution of $1280 \times 1024$ pixels (Philips Healthcare, Best, Netherlands).

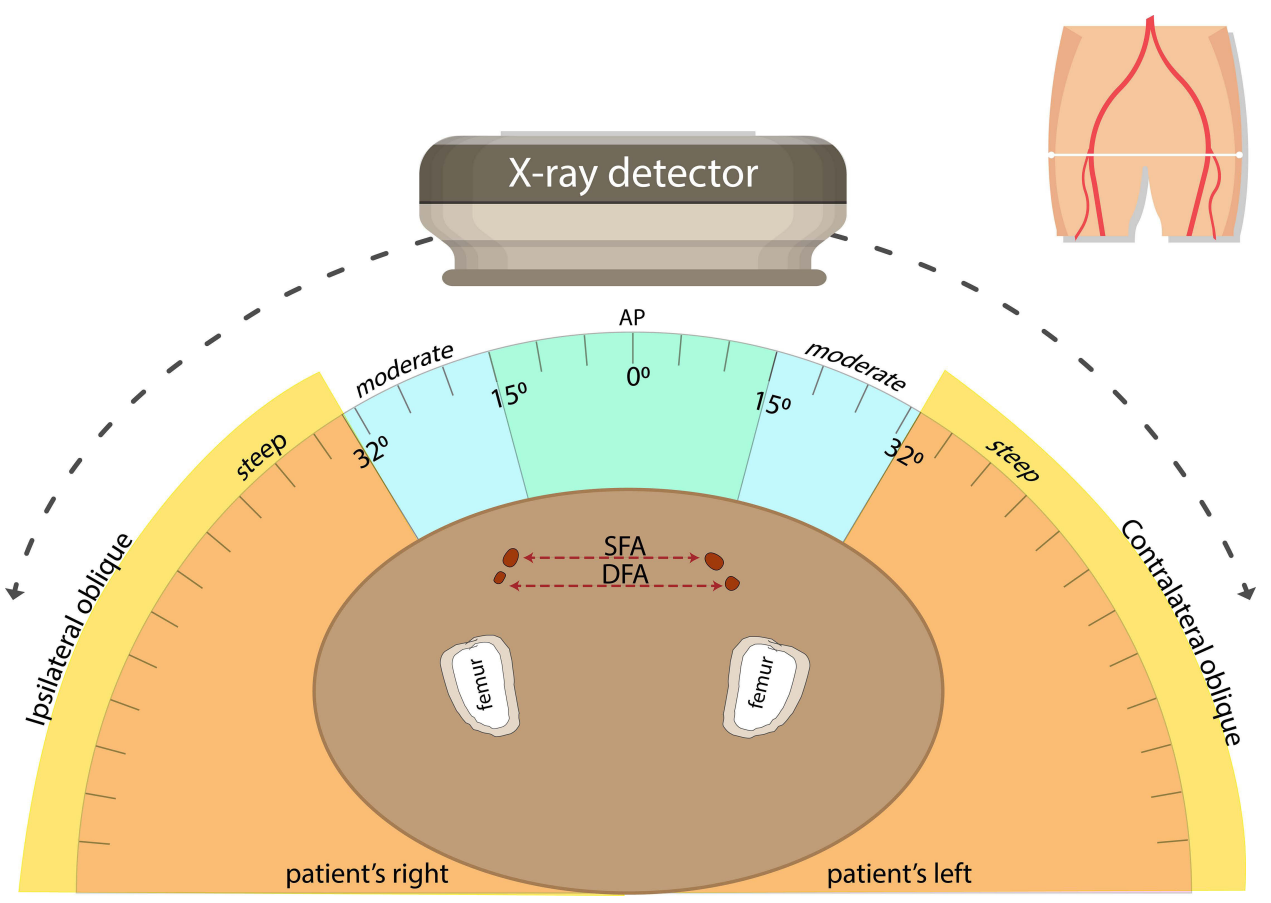

Figure 3 Categorization of tube angles in ipsilateral and contralateral views when visualising the right common femoral artery. Cross-sectional axial image representative of the level of the common femoral artery bifurcation. AP ranges from $-15^{\circ}$ to $+15^{\circ}$, moderate ranges from \pm 16 to $32^{\circ}$, and steep is $\geq 32^{\circ}$ angulation.

Abbreviations: AP, anterior-posterior; DFA, deep femoral artery; SFA, superficial femoral artery. 
The sample included imaging with fluoro save (35\%), cine angiography (48\%), and DSA (16\%). Images were purposely chosen from cases with a range of patient BMIs from 16 to 55 (mean-29) to reflect a typical patient cohort. If two or more CFA acquisitions were taken during a single procedure, customarily the RAO and the LAO views, all images were provided to the observer. Images that were non-diagnostic due to factors such as poor contrast opacification or patient movement were excluded from the sample.

A standardized questionnaire was modified from the one used for similar purposes by Walsh et $\mathrm{al}^{17}$ and included quantitative and qualitative responses (supple mental document 1). Questions included judgment on acquisition mode, if the image was clinically fit for purpose, and whether there was identifiable pathology. A question was included which asked the observer whether they felt an acquisition mode of lesser quality would have sufficed. Training was provided in image evaluation and questionnaire format. Questionnaires were completed independently on an identical image dataset, and no inter-observer consultation occurred. The observer results were correlated to determine whether higher dose modes of acquisition were clinically justifiable.

Ethics approval for the image quality assessment was obtained from Ramsay Human Research Ethics Committee (Protocol number-20/02).

\section{Statistical Analysis}

Temple staff doses (to the doctor, scrub nurse, and scout nurse) were approximately log-normally distributed, and therefore were $\log$ transformed for the analyses. Other variables, such as AK, DAP, average number of cine acquisitions performed per case, average volume of contrast medium, cine or fluoroscopy time, were similarly lognormally distributed and thus log transformed for the analyses.

Aim 1: Imaging modes were summarized for patient and staff doses using geometric means and 95\% confidence intervals (CIs).

Aims 1 and 2: To investigate the influence of the CFA imaging mode on patient and staff doses, we used linear regression (with log-normal distribution for staff doses) with backward elimination. Imaging modes (variable of interest) and all candidate variables with $p \leq 0.25$ in the univariate analyses were included in the initial multivariable model. Candidate variables were then sequentially removed if they were both nonsignificant and not a confounder. Confounding was evaluated as at least $20 \%$ change in any remaining parameter estimates as compared to the full model, and variables were considered significant in the final model if the $p \leq 0.10$. Results of the linear regression were reported as the ratio of geometric mean of staff doses for the category of interest compared to the reference.

Aim 2: Additionally, the effect of femoral imaging TA on patient dose was summarised using geometric means with $95 \%$ CIs.

Aim 3: To evaluate whether lower dose modes provide images of sufficient clinical quality to assess suitability for VCD deployment and diagnose pathology, we fitted a random-effects logistic model for repeated measures. Results from the random-effects logistic model were reported as odds ratios (ORs) and 95\% CIs. STATA version 15.1 (Stata Corporation, College Station, Texas, USA) and Statistical Discovery Software JMP Pro (Version 15.2.0 SAS Institute, Cary, NC, USA) were used for all analyses, while Adobe illustrator (Version 25.1, San Jose, California, USA) and Microsoft excel (Redmond, Washington, USA) were used for image development.

\section{Results}

\section{Patient and Staff Dose}

Patient and staff dose data were collected for femorally accessed procedures $(\mathrm{n}=782)$ which did not employ CFA imaging, utilized fluoro save, cine angiography, or DSA (Table 1). There was no significant association between staff dose and the inclusion or mode of femoral imaging, as shown by the overlapping CIs. A comparison of mean basic procedural information for each imaging category is presented in Table 2.

In the linear regression models, larger patient BMIs $(\mathrm{p}<0.001)$, as well as AK $(\mathrm{p}<0.001)$, DAP $(\mathrm{p}<0.001)$, scrub dose $(p<0.001)$, scout dose $(p=0.010)$, number of cine runs $(p=0.001)$, contrast volume $(p=0.040)$ and fluoroscopy $(\mathrm{p}=0.003)$ were all independently associated with greater dose to the doctor (dr) (data are not shown). Stenting of plaques of greater complexity was also found to be related to a higher dose to $\operatorname{Dr}(\mathrm{p}=0.043)$, and this association remained significant even after adjusting for room ID, CFA imaging acquisition mode, number of CFA images taken, DAP, and scrub dose. 
Table I Patient and Staff Dose Measurements for Differing Femoral Artery Imaging Modes

\begin{tabular}{|c|c|c|c|c|c|c|c|c|c|}
\hline \multirow[t]{2}{*}{ Acquisitions } & \multicolumn{3}{|c|}{ Patient Dose } & \multicolumn{6}{|c|}{ Temple Dose to Staff } \\
\hline & $\begin{array}{l}\text { No of } \\
\text { Proc }\end{array}$ & DAP (Gy-cm2) & AK (Gy) & $\begin{array}{l}\text { No of } \\
\text { Proc }\end{array}$ & Doctor $(\mu \mathrm{Sv})$ & $\begin{array}{l}\text { No of } \\
\text { Proc }\end{array}$ & $\begin{array}{l}\text { Scrub Nurse } \\
(\mu \mathrm{Sv})\end{array}$ & $\begin{array}{l}\text { No of } \\
\text { Proc }\end{array}$ & $\begin{array}{l}\text { Scout } \\
\text { Nurse }(\mu S v)\end{array}$ \\
\hline No imaging & 227 & $20.3(18.4,22.4)$ & $0.4(0.3,0.4)$ & 156 & $0.8(0.6,1.0)$ & 164 & I $(0.8, \mid .2)$ & 83 & $0.2(0.1,0.2)$ \\
\hline Fluoro save & 129 & I8.3 (I6.I, 20.9) & $0.3(0.3,0.4)$ & 86 & $0.7(0.5,1.0)$ & 97 & $1.14(0.9,1.5)$ & 38 & $0.2(0.1,0.3)$ \\
\hline Cine & 409 & $19.3(18,20.8)$ & $0.4(0.3,0.4)$ & 323 & $0.9(0.8,1.1)$ & 273 & I.2 (I.I, I.5) & 140 & $0.1(0.1,0.2)$ \\
\hline DSA & 17 & $47.5(33.3,67.7)$ & $0.8(0.6,1.2)$ & 14 & I $(0.5,2.4)$ & II & I.6 $(0.7,3.7)$ & 10 & $0.3(0.1,0.8)$ \\
\hline
\end{tabular}

Notes: Values are geometric mean ( $95 \%$ confidence interval).

Abbreviations: AK, air kerma; DAP, dose area product; DSA, digital subtraction angiography; Gy, gray; Gy-cm², gray-centimetres squared; No, number; proc, procedures; $\mu$ Sv, microSievert.

Table 2 Procedural Parameters for Differing Femoral Artery Imaging Modes

\begin{tabular}{|l|c|c|c|c|}
\hline Acquisitions & Cine/Fluoro Time & Cine Runs & Contrast (mIs) & Pt BMI \\
\hline No imaging $(n=227)$ & $3.3(2.9,3.7)$ & $10.4(9.8,11.0)$ & $92.4(86.2,99.1)$ & $28.9(28.2,29.6)$ \\
Fluoro save $(n=129)$ & $3.7(3.15,4.3)$ & $11.7(10.8,12.8)$ & $99.4(90.5,109)$ & $29.6(28.7,30.5)$ \\
Cine $(n=409)$ & $4.11(3.8,4.5)$ & $12.9(12.3,13.5)$ & $98.2(93.2,103.5)$ & $29.1(28.6,26.7)$ \\
DSA $(n=17)$ & $10.8(7.1,16.3)$ & $21(16.7,26.4)$ & $124.4(96.3,160.7)$ & $29(26.5,31.5)$ \\
\hline
\end{tabular}

Notes: Values for the procedural parameters are geometric mean ( $95 \%$ confidence interval) and the mean for patient BMI ( $95 \%$ confidence interval).

Abbreviations: BMI, body mass index; Fluoro, fluoroscopy; DSA, digital subtraction angiography; mins, minutes; mls, millilitres; Pt, patient.

Similarly, increasing patient BMI $(\mathrm{p}=0.002)$, and scout dose $(\mathrm{p}=0.008)$, AK $(\mathrm{p}<0.001)$, DAP, and Dr dose $(p<0.001)$ were independently associated with a higher dose to the scrub nurse, and this association remained significant even after adjusting for the same variables as for the dose to $\mathrm{dr}$ as well as adjusting for the procedure type, whether it was performed by an interventionalist, included CFA imaging, and dr dose (instead of scrub dose). Procedures performed by an interventionalist when compared to a noninterventionalist were found to be inversely associated with scrub dose $(\mathrm{p}<0.001)$ and, to a lesser extent, with scout dose $(p=0.053)$. For the scrub dose, this association remained significant even after adjustment. There was no observed association between interventionalist/ non-interventionalist and $\mathrm{dr}$ dose.

The procedures which included intervention were associated with greater dose to the scout nurse $(p<0.001)$, but not to the dr or scrub nurse, and as the number of plaques treated increased, so did the relative dose to the scout nurse $(p<0.001)$. The room in which the procedure was performed was also related to the levels of dose to the doctor $(p=0.002)$, scrub nurse $(p<0.001)$, and scout nurse $(\mathrm{p}=0.053)$, with the highest dose recorded for procedures performed in the room without the dose-reducing software but this was no longer significant after adjusting for room ID, CFA imaging acquisition mode, DAP, the total procedural fluoroscopy time and contrast volume, and the dose to the dr and scrub nurse.

\section{Patient Dose per Discrete CFA Imaging Event}

A comparison of patient dose during fluoroscopy, cine angiography, and DSA acquisitions demonstrated a significant difference between the modalities employed (Figure 4).

Unsurprisingly, we observed significantly increased levels of radiation exposure to be associated with the use of DSA, while the use of fluoro save was related to the least patient exposure of all three acquisition modes (Figure 4). Imaging in the contralateral angle (CL) was associated with an increased dose area product (DAP) and air kerma (AK) when compared with ipsilateral (IP) and anterior-posterior (AP) tube orientation.

The degree of TA was also found to affect patient dose with steeper tube angles associated with higher patient dose when using cine angiography or DSA. Table 3 demonstrates the effect of tube angle on patient dose when imaging CFA, while Table 4 illustrates the number of CFA acquisitions and frequency of use of various tube angulations. Right CFA access accounted for $88 \%$ of the included cases. 'Multiple acquisitions' refers to when more than one CFA imaging event occurred in a procedure and may reflect either that a number 


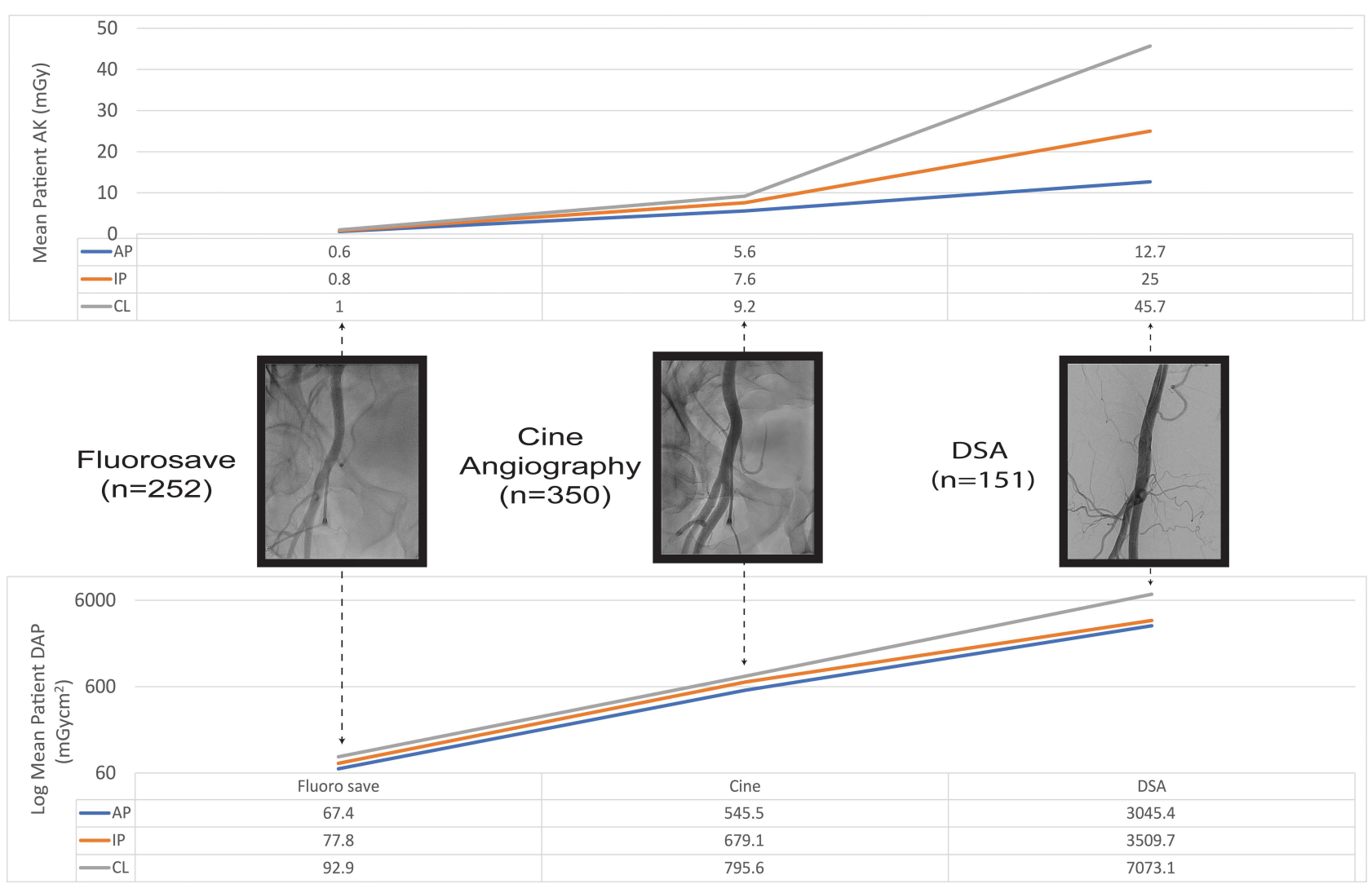

Figure $4 \mathrm{~A}$ comparison of patient dose area product (DAP) and air kerma (AK) when using fluorosave, cine angiography, and digital subtraction angiography (DSA) using the anterior-posterior (AP), ipsilateral, or contralateral tube angle.

of acquisitions were performed using a set tube angle, or multiple tube angles.

\section{Image Quality}

Observers selected which modality they surmised had been used to image the CFA and whether the image quality was sufficient for an experienced operator to be confident in diagnosing potential pathology. Responses to qualitative questions were minimal. Questionnaire results are presented in table 5.

Given the potential effect of patient BMI on image noise, random-effects logistic regression for repeated measures was performed to ascertain whether patient BMI potentially influenced observer selections. There was an interaction (although not significant at the 10\% level) that suggests that for patients with higher BMIs, the observers were less likely to choose cine when compared with fluoroscopy (data are not shown). No significant association was found between patient BMI and the correct identification of the mode of acquisition. Figure 5 provides example images for a range of patient BMIs and acquisition types. One observer was found to have significantly higher rates of identifying that a run of lesser dose would suffice when compared to the other. Only $1 \%$ of images were considered non-diagnostic by the observers, and it

Table 3 The Effect of Tube Angle on Patient Dose

\begin{tabular}{|l|c|c|c|c|c|c|}
\hline \multirow{2}{*}{ Acquisitions $^{\mathbf{a}}$} & \multicolumn{3}{|c|}{ Average DAP (Gy-cm2) } & \multicolumn{3}{c|}{ Average AK (Gy) } \\
\cline { 2 - 7 } & AP & Moderate & Steep & AP & Moderate & Steep \\
\hline Fluoro save (n=252) & $67.4(49.0,92.9)$ & $87(81.0,93.5)$ & $71.4(56.3,90.6)$ & $0.57(0.4,0.9)$ & $0.9(0.9,1.0)$ & $0.8(0.6,1.0)$ \\
Cine $(\mathrm{n}=351)$ & $545.5(400.1,743.7)$ & $722.4(678.5,769.0)$ & $925.78(788.6,1086.7)$ & $5.6(3.8,8.2)$ & $8.3(7.8,8.9)$ & $10.2(8.6,1.0)$ \\
DSA ( $\mathrm{n}=151)$ & $3045.4(2626.0,3531.8)$ & $3434.8(3037.1,3884.5)$ & $6156(4111.3,9217.8)$ & $12.7(10.5,15.3)$ & $23.2(20.4,26.4)$ & $63.1(41.8,95.2)$ \\
\hline
\end{tabular}

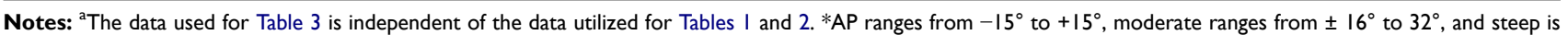
$\geq 32^{\circ}$ angulation. Values for the DAP and AK are geometric mean ( $95 \%$ confidence interval).

Abbreviations: AK, air kerma; AP, anterior-posterior; DAP, dose area product; DSA, digital subtraction angiography; Gy, gray; Gy-cm², gray-centimetres squared. 
Table 4 Number and Angle of Common Femoral Artery Acquisitions Performed per Case

\begin{tabular}{|l|l|c|c|}
\hline $\begin{array}{l}\text { Imaging of the } \\
\text { Femoral Artery }\end{array}$ & & Number & Percentage \\
\hline Number of & Multiple $^{\mathrm{b}}$ & 178 & 32.7 \\
acquisitions $^{\mathrm{a}}$ & Single & 367 & 67.3 \\
\hline Total & & 545 & 100 \\
\hline Imaging tube & Both obliques & 130 & 23.9 \\
angles & Ipsilateral only & 143 & 26.2 \\
& Contralateral only & 202 & 37.1 \\
& AP only & 49 & 9.0 \\
& Oblique and AP & 21 & 3.9 \\
\hline Total & & 545 & 100 \\
\hline
\end{tabular}

Notes: ${ }^{a}$ The data used for Table 4 is independent of the data utilized for Tables I and 2. "Multiple" refers to more than one acquisition performed either in the same tube angle, or using different tube angles. AP, anterior-posterior *oblique refers to ipsilateral or contralateral angulation.

Table 5 Number of Runs per Each Imaging Type Correctly Identified by the Observers

\begin{tabular}{|l|l|c|c|}
\hline \multirow{2}{*}{ Imaging Type } & \multirow{2}{*}{ Total } & \multicolumn{2}{|c|}{ Correct } \\
\cline { 3 - 4 } & & No n (\%) & Yes n (\%) \\
\hline Fluoro save & $92(35.0)$ & $19(20.6)$ & $73(79.4)$ \\
Cine & $127(48.3)$ & $30(23.6)$ & $97(76.4)$ \\
DSA & $44(16.7)$ & $0(0)$ & $44(100.0)$ \\
Total & $263(100.0)$ & $49(18.6)$ & $214(81.4)$ \\
\hline
\end{tabular}

Notes: Values are numbers of correctly identified CFA images per modality (percentage in the acquisition category). ${ }^{*}$ Chi-squared $\mathrm{P}$-value $=0.002$.

was deemed that a run of lesser dose would be sufficient in $78 \%$ of cases.

\section{Discussion}

This study demonstrated that utilizing higher dose modes for imaging the femoral artery access point during coronary angiography is associated with an increased dose to the patient. Using fluoro save provides adequate clinical detail while exposing the patient to minimum dose, thus adhering to the ALARA principle. Steeper tube angles and imaging in the contralateral oblique are related to higher patient dose.

\section{Femoral Imaging During Coronary Angiography -Implications for Patients}

The effect of femoral imaging modality on patient dose parameters of AK and DAP was investigated in this study. Both are useful parameters to determine the potential detrimental effects of radiation on the patient. It was demonstrated that DSA imaging of the CFA was associated with significantly higher patient DAP and AK when compared to cine or fluoro save.

The need for imaging the CFA with DSA is contentious. The arguments against this include increased radiation dose, the inability to visualize bony anatomy, and the potential to miss a small vessel leak due to subtraction. Given the higher tube output required for DSA, it is unsurprising that this study's results indicate a significant increase in patient DAP and AK. These findings support those of Zurcher et al, who reported a patient dose reduction of $40 \%$ when DSA was substituted by fluoroscopic imaging during endovascular aneurysm repairs, ${ }^{18}$ and Pearl et al who concluded that DSA of the femoral artery should be replaced with fluoro save during cerebral angiography. ${ }^{15}$

Ipsilateral imaging (ie the RAO for the right CFA) is considered to be the best orientation to display the bifurcation of the deep and superficial femoral branches, ${ }^{19}$ but this view was used exclusively in only $26 \%$ of included cases. CFA imaging performed in the AP view $\left(\leq 15^{\circ}\right)$ was associated with significantly lower radiation dose to the patient, but imaging in the AP view only may lead to misdiagnosis. ${ }^{20}$ Utilizing tube angles $\geq 32^{\circ}$ increases patient dose and has also been shown to increase staff dose, especially in the LAO view. ${ }^{14}$ Given that performing the contralateral view is associated with an increase in patient dose, operators should consider primarily using the ipsilateral view to image the right CFA, and supplement this with an LAO only if further imaging is required to visualize the bifurcation and sheath insertion point better.

\section{Femoral Imaging During Coronary Angiography - Implications for Staff}

Since the staff dose had a strong relationship with DAP and $\mathrm{AK}$, the association between staff dose and other variables, such as the inclusion of femoral imaging, was difficult to ascertain.

Due to the surprising association of higher dose levels in the "no imaging" dataset, when compared with fluoro save, further analysis was performed to identify potential contributing factors. It was postulated that a higher number of interventional procedures in the cases that did not image the CFA might have contributed to the higher-thanexpected dose for this group, but each set was composed of approximately $20 \%$ interventional procedures, except in 


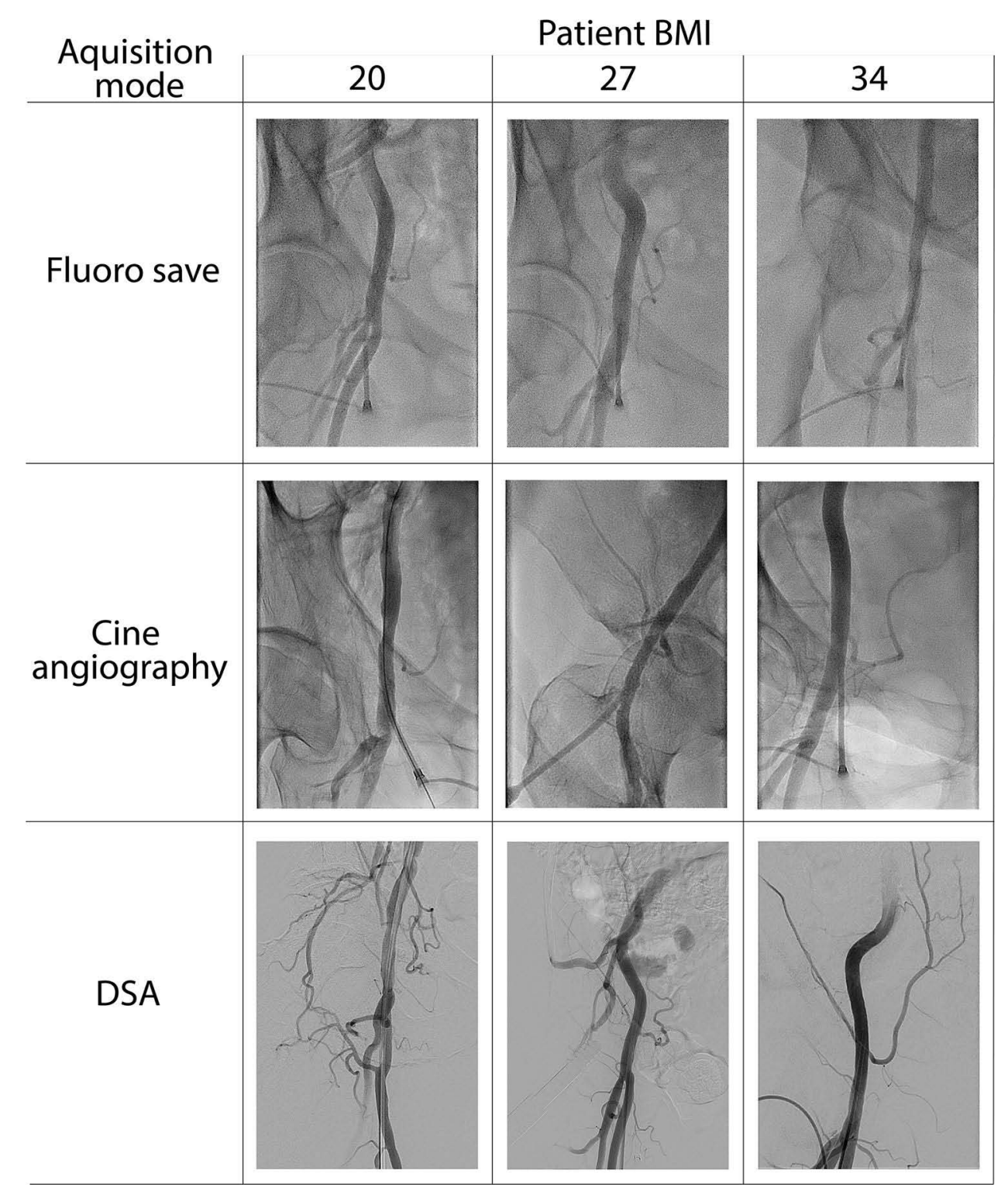

Figure 5 Examples of images of the common femoral artery using fluoro save, cine angiography and digital subtraction angiography (DSA) in patients of differing body mass index (BMI).

cases imaging the CFA by DSA (65\%). Another consideration was the potential dose impact when multiple femoral acquisitions were taken within a single case, but it was also found that this occurred at a similar frequency across the acquisition modes.

Despite being unable to demonstrate a significant association between higher dose imaging modes and staff dose, the observed correlation to increased patient dose does have practical clinical implications for staff. An increase in patient dose will inevitably lead to an increase in staff dose. $^{12,21}$ It has been previously reported that scrub or assistant dose is lower than that of the cardiologist (table $6),{ }^{22}$ but this study has demonstrated a higher average dose received by the scrub nurse compared to the cardiologist, confirming the previous results published by our group. ${ }^{23}$ This is postulated to be a result of the positioning of the ceiling-mounted lead directly in front of the cardiologist, providing a protective effect for them, but not staff adjacent to them. ${ }^{23}$ Given the greater number of procedures, a nurse is typically involved in compared with a dr, this is cause for concern. Procedures performed on the angiographic system without the dose reducing software was associated with a significant increase in dose to staff, which has been demonstrated previously. ${ }^{24}$ It is worth noting that the levels of patient exposure measured in this study were similar or lower than those reported in similar studies (table 6) with the 
Table 6 Comparison of Staff and Patient Dose for Femorally Accessed Coronary Angiography

\begin{tabular}{|c|c|c|c|c|c|c|c|c|c|}
\hline Author & $\begin{array}{c}\text { Year } \\
\text { Published }\end{array}$ & Procedure & $\begin{array}{l}\text { Acquisition } \\
\text { Mode }\end{array}$ & Number & $\begin{array}{c}\text { Cardiologist } \\
(\mu \mathrm{Sv})\end{array}$ & $\begin{array}{c}\text { Scrub/ } \\
\text { Assistant }(\mu \mathrm{Sv})\end{array}$ & $\begin{array}{l}\text { Scout } \\
(\mu \mathrm{Sv})\end{array}$ & $\begin{array}{c}\text { DAP } \\
\left(\mathrm{cGy} \cdot \mathrm{cm}^{2}\right)\end{array}$ & $\begin{array}{c}\text { AK } \\
(m G y)\end{array}$ \\
\hline \multirow[t]{4}{*}{$\begin{array}{l}\text { Wilson-Stewart } \\
\text { et al. }\end{array}$} & & \multirow[t]{4}{*}{$\mathrm{CA}$ and $\mathrm{PCl}$} & $\begin{array}{l}\text { No CFA } \\
\text { imaging }\end{array}$ & 227 & $0.77^{\mathrm{a}}$ & $1.00^{\mathrm{a}}$ & $0.17^{\mathrm{a}}$ & 2032 & 380 \\
\hline & & & Fluorosave & 129 & $0.68^{\mathrm{a}}$ & $1.14^{\mathrm{a}}$ & $0.17^{\mathrm{a}}$ & 1833 & 340 \\
\hline & & & Cine & 409 & $0.89^{\mathrm{a}}$ & $1.24^{\mathrm{a}}$ & $0.14^{\mathrm{a}}$ & 1933 & 370 \\
\hline & & & DSA & 17 & $1.04^{\mathrm{a}}$ & $1.61^{\mathrm{a}}$ & $0.31^{\mathrm{a}}$ & 4750 & 810 \\
\hline \multirow[t]{2}{*}{ Kim et $\mathrm{al}^{22}$} & \multirow[t]{2}{*}{2020} & $C A$ & & & $10.10^{\mathrm{bc}}$ & $3.592^{\mathrm{bc}}$ & & 4695 & \\
\hline & & $\mathrm{PCl}$ & & & $42.89^{\mathrm{bc}}$ & $11.30^{\mathrm{bc}}$ & & 15,465 & \\
\hline Tarighatnia et al ${ }^{14}$ & 2017 & $\mathrm{CA}$ & & 101 & $8.37^{c}$ & & & 1513 & 195 \\
\hline Pancholy et $\mathrm{al}^{25}$ & 2015 & $\mathrm{CA}$ & & 498 & $20^{c}$ & & & 2540 & 421 \\
\hline \multirow[t]{2}{*}{ Rychlik et $\mathrm{a}^{26}$} & \multirow[t]{2}{*}{2018} & $\mathrm{CA}$ & & & & & & 3130 & \\
\hline & & $\mathrm{PCl}$ & & 1275 & & & & 6550 & \\
\hline \multirow[t]{3}{*}{ Ureyen et $\mathrm{al}^{27}$} & \multirow[t]{3}{*}{2017} & $\mathrm{CA}$ & & 856 & & & & 799 & 65 \\
\hline & & $\mathrm{PCl}^{\mathrm{d}}$ & & 407 & & & & 3006 & 259 \\
\hline & & $\mathrm{PCl}^{\mathrm{e}}$ & & 989 & & & & 3493 & 323 \\
\hline \multirow[t]{2}{*}{ Georges et $\mathrm{al}^{28}$} & \multirow[t]{2}{*}{2017} & CA & & 9055 & & & & 2810 & \\
\hline & & $\mathrm{PCl}$ & & 7865 & & & & 5940 & \\
\hline
\end{tabular}

Notes: CFA, common femoral artery; ${ }^{a}$ dosimeter worn on left temple; breported dose is a calculation of effective dose; 'badge worn external to lead over upper left chest; badge worn external to lead breast pocket; ${ }^{d}$ stable angina; ${ }^{e}$ acute coronary syndrome.

exception of Targhatnia et al, possibly due to the utilization of lower frame rates.

\section{Image Quality}

The image assessment component of this study included femoral imaging utilizing fluoroscopy, cine angiography, and DSA. A range of patient BMIs for each acquisition type was intentionally included as BMI has been shown to affect image quality. ${ }^{29}$

This study has shown that sufficient image quality can be obtained using lower dose imaging modes when assessing the CFA during coronary angiography. The dose reduction benefits of substituting cine acquisitions with fluoro save have been previously noted, with Leyton et al demonstrating that the scatter dose rate to the operator's eye region is increased by a factor of six when using cine acquisitions as opposed to fluoroscopy. ${ }^{30}$ As manufacturers continue to improve dose reduction techniques, and similar or better image quality can be achieved with less radiation dose, ${ }^{31}$ operators should consider routinely utilizing lower dose modes to reduce radiation dose to the patient, which will in turn lesson staff dose. ${ }^{12,21}$

\section{Limitations}

Image quality is affected by numerous procedural and patient characteristics; hence, in addition to subjective observer evaluation of image quality, it would be useful to include a quantitative evaluation. Given that the clinical premise of utilizing $\mathrm{x}$-ray imaging is to ascertain the presence of pathology or visualize anatomy, it is considered appropriate to focus on image quality in a clinically applicable form.

\section{Conclusion}

This study has demonstrated that the use of higher dose imaging of the femoral artery increases patient dose. It was found that fluoro save frequently provided adequate diagnostic quality for visualizing vascular access points and diagnosing pathology. DSA of the CFA should be avoided during transfemoral coronary angiograms unless critical to diagnostic analysis. Due to staff dose being highly correlated with DAP and AK, it is difficult to 
observe any association between staff dose and CFA imaging mode, but this does not discount an increased dose burden to the staff due to the use of cine angiography or DSA. Imaging of the CFA in the contralateral oblique and steeper tube angles increased patient dose. It is recommended that fluoroscopic operators consider utilizing lower dose modes in the ipsilateral orientation and avoiding steep x-ray tube angles to reduce the risk of radiation exposure with the new imaging mantra of 'acceptable, not impeccable.

\section{Abbreviations}

ALARA, as low as reasonably achievable; AK, air kerma; AP, anterior-posterior; BMI, body mass index; CFA, common femoral artery; CI, confidence interval; CL, contralateral; DAP, dose area product; Dr, doctor; DSA, digital subtraction angiography; IL, ipsilateral; LAO, left anterior oblique; mGy, milliGray; mGy.cm2, milliGray per square centimeter; No, number; RA, radial artery; RAO, right anterior oblique; TAVI, transcatheter aortic valve implantation; $\mathrm{VCD}$, vascular closure device; $\mu \mathrm{Sv}$, microSievert.

\section{Data Accessibility Statement}

All data referred to and underpinning this publication are openly available in the QUT Research Data Finder and can be found at https://researchdatafinder.qut.edu.au/display/ $\underline{\mathrm{n} 30521}$

\section{Acknowledgments}

We thank our nursing, radiography, and medical colleagues from the cardiovascular suites at Greenslopes Private Hospital for their contributions towards data collection during this research. The corresponding author would like to acknowledge Australian Government Research Training Program Scholarship.

\section{Disclosure}

The authors report no financial or non-financial competing interests in this work.

\section{References}

1. Hernández C, Durán A, Cortés MC. Lesiones oculares y radiación ionizante. Revista Colombiana de Cardiología. 2020;27:72-78. doi:10.1016/j.rccar.2019.09.004

2. Ison GR, Allahwala U, Weaver JC. Radiation Management in Coronary Angiography: Percutaneous Coronary Intervention for Chronic Total Occlusion at the Frontier. Heart Lung Circ. 2019;28 (10):1501-1509. doi:10.1016/j.hlc.2019.05.004
3. Boice JD, Held KD, Shore RE. Radiation epidemiology and health effects following low-level radiation exposure. J Radiol Prot. 2019;39(4):S14-S27.

4. Klein LW, Tra Y, Garratt KN, et al. Occupational health hazards of interventional cardiologists in the current decade: Results of the 2014 SCAI membership survey. Catheter Cardiovasc Interv. 2015;86 (5):913-924. doi:10.1002/ccd.25927

5. Roguin A. Radiation Hazards to Interventional Cardiologists: A Report on Increased Brain Tumors Among Physicians Working in the Cath Lab. SOLACI. Buenos Aires: Argentina; 2014.

6. Aristizábal JM. Riesgo cardiovascular relacionado con la radiación ionizante. Revista Colombiana de Cardiología. 2020;27:21-24. doi:10.1016/j.rccar.2019.11.001

7. Borghini A, Vecoli C, Piccaluga E, Guagliumi G, Picano E, Andreassi MG. Increased mitochondrial DNA4977-bp deletion in catheterization laboratory workers with long-term low-dose exposure to ionizing radiation. Eur J Prev Cardiol. 2019;26(9):976-984. doi:10.1177/2047487319831495

8. Coppeta L, Pietroiusti A, Neri A, et al. Risk of radiation-induced lens opacities among surgeons and interventional medical staff. Radiol Phys Technol. 2019;12(1):26-29. doi:10.1007/s12194-018-0487-9

9. Castle E, Rathod K, Guttmann O, et al. Routine use of fluoroscopic guidance and up-front femoral angiography results in reduced femoral complications in patients undergoing coronary angiographic procedures: an observational study using an Interrupted Time-Series analysis. Heart Vessels. 2019;34(3):419-426. doi:10.1007/s00380018-1266-6

10. Chhatriwalla AK, Lim M, Sorajja P. Arterial and venous access. In: Sorajja P, Lim M, Kern M, editors. Kern's Cardiac Catheterization Handbook. 2020:79-130.

11. Kern MJ, Seto AH, Forsberg M. Vascular Access. In: Kern M, Sorajja P, Lim M, editors. The Interventional Cardiac Catheterization Handbook. Philadelphia, PA, U.S.A: Elsevier; 2018:51-87.

12. Moscucci M. Grossman \& Baim's Cardiac Catheterization, Angiography, and Intervention. Philadelphia: Wolters Kluwer; 2015.

13. Schueler BA, Fetterly KA, Balter S. Radiation Safety During Cardiovascular Procedures. In: Topol E, Teirstein P, editors. Textbook of Interventional Cardiology. 8 ed. Philadelphia, PA, U.S. A: Elsevier; 2020:128-138.e122.

14. Tarighatnia A, Mohammadalian A, Ghojazade M, Pourafkari L, Farajollahi A. Beam projections and radiation exposure in transradial and transfemoral approaches during coronary angiography. Anatol J Cardiol. 2017;18(4):298-303. doi:10.14744/AnatolJCardiol.2017.7724

15. Pearl MS, Torok C, Wang J, Wyse E, Mahesh M, Gailloud P. Practical techniques for reducing radiation exposure during cerebral angiography procedures. J Neurointerv Surg. 2015;7(2):141. doi:10.1136/neurintsurg-2013-010982

16. Geber T, Gunnarsson M, Mattsson S. Eye lens dosimetry for interventional procedures - Relation between the absorbed dose to the lens and dose at measurement positions. Radiat Meas. 2011;46 (11):1248-1251. doi:10.1016/j.radmeas.2011.07.028

17. Walsh C, Dowling A, Meade A, Malone J. Subjective and objective measures of image quality in digital fluoroscopy. Radia Prot Dosimetry. 2005;117(1-3):34-37. doi:10.1093/rpd/nci708

18. Zurcher KS, Naidu SG, Money SR, et al. Dose Reduction Using Digital Fluoroscopy Versus Digital Subtraction Angiography in EVAR: A Prospective Randomized Trial. J Vasc Surg. 2020;72 (6):1938-1945. doi:10.1016/j.jvs.2020.02.050

19. Minici R, Paone S, Talarico M, et al. Percutaneous treatment of vascular access-site complications: a ten years' experience in two centres. CVIR Endovascular. 2020;3(1):29. doi:10.1186/s42155-020-00120-7

20. Rane N, Imam A, Foley P, Timmons G, Uberoi R. Pelvic digital subtraction catheter angiography-Are routine oblique projections necessary? Eur J Radiol. 2011;77(1):182-184. doi:10.1016/j.ejrad. 2009.07.008 
21. López PO, Dauer LT, Loose R, et al. ICRP Publication 139: Occupational Radiological Protection in Interventional Procedures. Annals of the ICRP. 2018;47(2):1-118. doi:10.1177/ 0146645317750356

22. Kim J-S, Lee B-K, Ryu D-R, et al. Occupational radiation exposure in femoral artery approach is higher than radial artery approach during coronary angiography or percutaneous coronary intervention. Sci Rep. 2020;10(1):7104. doi:10.1038/s41598-02062794-2

23. Wilson-Stewart K, Hartel G, Fontanarosa D. Occupational radiation exposure to the head is higher for scrub nurses than cardiologists during cardiac angiography. J Adv Nurs. 2019;75(11):2692-2700. doi:10.1111/jan.14085

24. Abuzeid W, Abunassar J, Leis JA, et al. Radiation safety in the cardiac catheterization lab: A time series quality improvement initiative. Cardiovasc Revasc Med. 2017;18(5,Supplement 1):S22-S26. doi:10.1016/j.carrev.2017. 04.009

25. Pancholy SB, Joshi P, Shah S, Rao SV, Bertrand OF, Patel TM. Effect of Vascular Access Site Choice on Radiation Exposure During Coronary Angiography: The REVERE Trial (Randomized Evaluation of Vascular Entry Site and Radiation Exposure). JACC Cardiovasc Interv. 2015;8(9):1189-1196. doi:10.1016/j.jcin.2015.03.026
26. Rychlik J, Hornacek I, Tejc M, Petrikovits E, Klimsa Z. Retrospective analysis of coronary interventions in a single centre and comparison of specific differences between radial and femoral access. Acta Cardiol. 2019;74(4):325-330. doi:10.1080/00015385.2018.1494115

27. Üreyen ÇM, Coşansu K, Vural MG, et al. Is trans-radial approach related to an increased risk of radiation exposure in patients who underwent diagnostic coronary angiography or percutaneous coronary intervention? (The SAKARYA study). Anatolian $j$ Cardiol. 2019;22(1):5-12. doi:10.14744/AnatolJCardiol.2019.06013

28. Georges J-L, Belle L, Meunier L, et al. Radial versus femoral access for coronary angiography and intervention is associated with lower patient radiation exposure in high-radial-volume centres: insights from the RAY'ACT-1 study. Arch Cardiovasc Dis. 2017;110 (3):179-187. doi:10.1016/j.acvd.2016.09.002

29. Reynolds, A. Obesity and Medical Imaging Challenges. Radiol Technol. 2011;82(3):219-239.

30. Leyton F, Nogueira MS, Gubolino LA, Pivetta MR, Ubeda C. Correlation between scatter radiation dose at height of operator's eye and dose to patient for different angiographic projections. Appl Radiat Isot. 2016;117:100-105. doi:10.1016/j.apradiso.2016.01.013

31. Taddeucci A, Piffer S, Redapi L. European Commission publication $\mathrm{RP}-162$ as a strong reference for reducing patient radiation dose: Results from an angiography equipment updating. Phys Med. 2020;71:132-136. doi:10.1016/j.ejmp.2020.02.019
Journal of Multidisciplinary Healthcare

\section{Publish your work in this journal}

The Journal of Multidisciplinary Healthcare is an international, peerreviewed open-access journal that aims to represent and publish research in healthcare areas delivered by practitioners of different disciplines. This includes studies and reviews conducted by multidisciplinary teams as well as research which evaluates the results or conduct of such teams or healthcare processes in general. The journal covers a very wide range of areas and welcomes submissions from practitioners at all levels, from all over the world. The manuscript management system is completely online and includes a very quick and fair peer-review system. Visit http://www.dovepress.com/testimonials. php to read real quotes from published authors. 